\title{
The Schrödinger equation on a compact manifold : Strichartz estimates and applications
}

\author{
Nicolas Burq Patrick Gérard Nikolay Tzvetkov \\ Résumé
}

Nous établissons des estimations de Strichartz avec perte de derivée fractionnaire pour l'équation de Schrödinger sur toute variété riemannienne compacte. Nous en deduisons des théorèmes d'existence globale pour le problème de Cauchy d'équations de Schrödinger non-linéaires sur les surfaces dans le cas de non-linéarités polynomiales défocalisantes, et sur les variétés de dimension trois dans le cas de non-linéarités quadratiques. Nous discutons également l'optimalité de ces estimées de Strichartz sur les sphères.

\begin{abstract}
We prove Strichartz estimates with fractional loss of derivatives for the Schrödinger equation on any riemannian compact manifold. As a consequence we infer global existence results for the Cauchy problem of nonlinear Schrödinger equations on surfaces in the case of defocusing polynomial nonlinearities, and on three-manifolds in the case of quadratic nonlinearities. We also discuss the optimality of these Strichartz estimates on spheres.
\end{abstract}

\section{Introduction}

Let $(M, g)$ be a complete riemannian manifold. The nonlinear Schrödinger equation on $M$ reads

$$
i \partial_{t} u+\Delta_{g} u=P^{\prime}\left(|u|^{2}\right) u, \quad u(0, x)=u_{0}(x) .
$$

where $\Delta_{g}$ denotes the Laplace-Beltrami operator on $M$, and $P: \mathbb{R}_{+} \rightarrow \mathbb{R}$ is a (at least $C^{1}$ ) function, for instance polynomial. It is classical that equation (1.1) satisfies the following formal conservation laws :

$$
\|u(t)\|_{L^{2}}=\left\|u_{0}\right\|_{L^{2}}
$$

We thank H. Koch and S. Zelditch for discussions about the optimality of our results on spheres and Zoll manifolds, and E. Fouvry and R. De la Bretèche for discussions about Gauss sums.

Keywords : Strichartz estimates, nonlinear Schrödinger, eigenfunctions, WKB methods, dispersive equations. 
and

$$
\int_{M}\left|\nabla_{g} u(t, x)\right|^{2} d x+\int_{M} P\left(|u(t, x)|^{2}\right) d x=E_{0} .
$$

Typical questions of interest about this equation are

(i) Global existence of strong solutions in the energy space $\left(E_{0}<+\infty\right)$ for defocusing nonlinearities (namely, $P \geq 0$, or more generally $E_{0}$ and $\left\|u_{0}\right\|_{L^{2}}$ control the $H^{1}$ norm of $u$ ).

(ii) Regularity of $u$ if $u_{0}$ and $P$ are regular.

(iii) Stability of particular global solutions.

(iv) Blow up of solutions for focusing nonlinearities (for instance $P(r)=-r^{m}$ for $m$ large enough).

Here we shall focus on the first two questions above. Observe that these problems are more involved than the corresponding ones for the wave equation. Indeed, equation (1.1) does not satisfy finite propagation speed, so the geometry of $M$ is expected to have a much stronger influence on the behavior of solutions. In the present work, we begin the systematic study of equation (1.1) in the particular case of a compact manifold. It turns out that our methods can also be applied to metrics on $\mathbb{R}^{d}$ with uniformity assumptions at infinity. Before stating our results, let us recall briefly what is known in the two cases which were already studied in the literature, namely the standard flat metrics on $\mathbb{R}^{d}$ and on $\mathbb{T}^{d}$.

\subsection{The case of $\mathbb{R}^{d}$ with the standard metric.}

If $d=1$, the Sobolev imbedding $H^{1}(\mathbb{R}) \subset L^{\infty}(\mathbb{R})$ and standard $H^{s}$ estimates for $\mathrm{e}^{i t \Delta}$ imply easily global existence of finite energy solutions for defocusing nonlinearity $P$, as well as regularity if $P$ is regular. The situation is more intricate in two space dimensions, since in this case $H^{1}\left(\mathbb{R}^{2}\right) \not \subset L^{\infty}\left(\mathbb{R}^{2}\right)$. However, the criticality of the latter imbedding is expressed by the following logarithmic estimate,

$$
\|u\|_{L^{\infty}} \leq C\|u\|_{H^{1}}\left(\log \left(2+\frac{\|u\|_{H^{2}}}{\|u\|_{H^{1}}}\right)\right)^{1 / 2} .
$$

Using this estimate, Brézis and Gallouët proved in [6] the global existence of smooth $\left(H^{2}\right)$ solutions in the case of a defocusing cubic nonlinearity $\left(P(r)=r^{2}\right)$. It should be observed that this approach holds as well on compact surfaces or on bounded plane domains, as stressed in [6]. However, even in $\mathbb{R}^{2}$ it does not yield global existence of $H^{1}$ solutions, nor does it allow to handle higher-degree nonlinearities. This program has been developed for about twenty years by many authors, including Ginibre-Velo [12],[13], Kato [18], Yajima [30], Cazenave [8] and Cazenave-Weissler [9], by proving and using the following inequalities, named after Strichartz in reference to the paper [28]. Given $v_{0} \in L^{2}\left(\mathbb{R}^{d}\right)$, set

$$
v(t, x)=\mathrm{e}^{i t \Delta} v_{0}(x) .
$$


Then, for every pair $(p, q)$ satisfying

$$
\frac{2}{p}+\frac{d}{q}=\frac{d}{2}, \quad p \geq 2, \quad(p, q) \neq(2, \infty)
$$

we have

$$
\|v\|_{L^{p}\left(\mathbb{R}, L^{q}\left(\mathbb{R}^{d}\right)\right)} \leq C\left\|v_{0}\right\|_{L^{2}\left(\mathbb{R}^{d}\right)} .
$$

Notice that the endpoint above estimates corresponding to $p=2, q=2^{*}$ if $d \geq 3$ were obtained recently by Keel and Tao [19]. These inequalities lead for instance to global existence and regularity of energy solutions to (1.1) on $\mathbb{R}^{d}$ for any defocusing polynomial nonlinearity if $d=2$, and for any defocusing subquintic nonlinearity if $d=3$. The quintic nonlinearity was solved in the case of three-dimensional radial data by Bourgain [5], while the case of general data is still open.

The key of inequalities (1.5) lies in the following estimate

$$
\left\|\mathrm{e}^{i t \Delta}\right\|_{L^{1}\left(\mathbb{R}^{d}\right) \rightarrow L^{\infty}\left(\mathbb{R}^{d}\right)} \leq \frac{C}{|t|^{d / 2}}
$$

which expresses the dispersive property of the linear Schrödinger equation on $\mathbb{R}^{d}$. Notice that this inequality is immediate from the explicit formula

$$
\mathrm{e}^{i t \Delta} v_{0}(x)=\frac{1}{(4 i \pi t)^{d / 2}} \int_{\mathbb{R}^{d}} \mathrm{e}^{i \frac{|x-y|^{2}}{4 t}} f(y) d y .
$$

The path from the dispersion estimate (1.6) to the Strichartz estimates (1.5) is now classical through a functional-analytic argument which comes back to Tomas [29] in the context of the related problem of restrictions of Fourier transforms (see Keel-Tao [19] for an abstract presentation of this argument).

\subsection{The case of flat tori.}

If $\mathbb{R}^{d}$ is replaced by a compact manifold $M$, the analogue of dispersion estimate (1.6) fails globally in time, as the example of constant solutions shows. It is less obvious, but still true, that it also fails locally in time (see the discussionin the beginning of section 3 below). In [2], [3] (see also [4]) Bourgain proposed a different approach to Strichartz estimates on the flat torus $M=\mathbb{T}^{d}$, based on the Fourier series representation of the Schrödinger group,

$$
v(t, x)=\mathrm{e}^{i t \Delta} v_{0}(x)=\sum_{k \in \mathbb{Z}^{d}} \mathrm{e}^{-i t|k|^{2}+k \cdot x} \hat{v}_{0}(k) .
$$

Notice that this representation shows that the solution is also periodic in time. By computing explicitly

$$
\|v\|_{L^{4}\left(\mathbb{T} \times \mathbb{T}^{d}\right)}^{4}=\left\|v^{2}\right\|_{L^{2}\left(\mathbb{T} \times \mathbb{T}^{d}\right)}^{2}
$$

by means of the Parseval identity, Bourgain obtains the following estimates.

(i) If $d=2,\|v\|_{L^{4}\left(\mathbb{T} \times \mathbb{T}^{2}\right)} \leq C_{\varepsilon}\left\|v_{0}\right\|_{H^{\varepsilon}\left(\mathbb{T}^{2}\right)}$ for all $\varepsilon>0$. By interpolating with Sobolev inequalities, this implies global existence for equation (1.1) with any polynomial defocusing nonlinearity. 
(ii) If $d \geq 3$,

$$
\|v\|_{L^{4}\left(\mathbb{T} \times \mathbb{T}^{d}\right)} \leq C\left\|v_{0}\right\|_{H^{s}\left(\mathbb{T}^{d}\right)}, \quad s>\frac{d}{4}-\frac{1}{2} .
$$

Unfortunately, even if $d=3$, this estimate is not sufficient to prove global existence results for nonlinear equations (it corresponds to a gain of $\frac{1}{2}-\varepsilon$ derivative with respect to the Sobolev imbedding, hence it only implies local wellposedness in $H^{1+\varepsilon}$ ). However, by proving more precise conormal estimates on nonlinear terms, Bourgain is able to derive global existence results : for instance, if $d=3$, he obtains global wellposedness for subquintic nonlinearities, the same result as in $\mathbb{R}^{3}$.

The goal of this work is to extend such results to more general compact manifolds.

\section{Statement of the results}

We begin with a generalization of Strichartz estimates.

Theorem 1. Let $(M, g)$ be a riemannian compact manifold of dimension $d \geq 1$ and $\Delta$ be the Laplace Beltrami operator on $M$. Given p, q satisfying the scaling condition $2 / p+d / q=d / 2$ and $p \geq 2, q<\infty$, the solution $v$ of (1.1), satisfies, for any finite time interval $I$,

$$
\|v\|_{L^{p}\left(I, L^{q}(M)\right)} \leq C(I)\left\|v_{0}\right\|_{H^{1 / p}(M)} .
$$

Remark 2.1. 1. Notice that the loss of $1 / p$ derivative in estimate (2.1) above is half of the loss of derivative which is predicted by the Sobolev imbedding $H^{s} \subset L^{q}$.

2. Theorem 1 can be easily generalized if one replaces $\Delta$ by any elliptic selfadjoint operator $P$ of order $m$, assuming that the vertical Hessian of the principal symbol of $P$ does not vanish outside the null section.

3. Other generalizations (abstract lower order perturbations, Riemannian metrics on $\mathbb{R}^{d}$ ) are given in section 4 below.

As a consequence, we infer a general result on nonlinear Schrödinger equations in dimension $d=2$ (see section 5 below for local well-posedness for equation (2.2) in more singular Sobolev spaces and arbitrary space dimensions).

Theorem 2. Let $(M, g)$ be a riemannian compact surface, and let $P$ be a polynomial function with real coefficients. For every $u_{0} \in H^{1}(M)$, there exists a unique maximal solution $u \in C\left(I, H^{1}(M)\right)$ of the equation

$$
i \partial_{t} u+\Delta u=P^{\prime}\left(|u|^{2}\right) u, \quad u(0, x)=u_{0}(x) .
$$

Moreover we have the following additional properties:

(i) If $\left\|u_{0}\right\|_{H^{1}(M)}$ is bounded from above, the length of $I \cap \mathbb{R}_{ \pm}$is bounded from below by a positive constant.

(ii) For any finite $p, u \in L_{l o c}^{p}\left(I, L^{\infty}(M)\right)$.

(iii) If $P(r) \longrightarrow+\infty$ as $r \longrightarrow+\infty, I=\mathbb{R}$. 
(iv) If $u_{0} \in H^{s}(M)$ for some $s>1, u \in C\left(I, H^{s}(M)\right)$. In particular if $u_{0} \in$ $C^{\infty}(M), u \in C^{\infty}(I \times M)$.

Remark 2.2. 1. If $P(r) \rightarrow+\infty$ when $r \rightarrow+\infty$, the existence of global weak solutions in $H^{1}$ is easy. A consequence of our estimates is that such solutions satisfy property (ii)and therefore are strong and uniquely determined by their Cauchy data.

2. Theorem 2 and Remark 2.1 can be extended with very slight modifications to other kinds of nonlinearities (for instance $P \in S^{m}(\mathbb{R}), P(r)=\lambda r^{\sigma+1}$ ).

In three space dimensions, we are unable to deduce global existence of strong $H^{1}$ solutions in the defocusing case from Theorem 1. However our methods can be used to prove the following three-dimensional analogue of Brézis-Gallouët's theorem.

Theorem 3. Assume $M$ is three-dimensional. Given $P \in S^{3 / 2}(\mathbb{R})$, for any $u_{0} \in$ $H^{2}(M)$, there exists a unique global solution $u \in C\left(\mathbb{R} ; H^{2}(M)\right)$ to the quadratic nonlinear Schrödinger equation

$$
i \partial_{t} u+\Delta u=P^{\prime}\left(|u|^{2}\right) u, \quad u(0, x)=u_{0}(x) .
$$

Let us come back to Theorem 1. On $(M, g)$ a natural generalization of Fourier series expansions is of course spectral decomposition of Laplace-Beltrami operator. However our knowledge of the spectrum of this operator on arbitrary riemannian manifolds is too poor for trying to adapt Bourgain's method at this level of generality. Rather, our proof of inequalities (2.1) relies on families of dispersive estimates on small time intervals depending on the size of the frequencies of the data. The strategy for proving such estimates is based on a simple time rescaling, first used by Lebeau [20] in the context of control theory, which allows us to use WKB construction of semiclassical parametrices. These WKB constructions are to be related to those used by Kapitanski [17] for proving Strichartz inequalities for wave equations with variable coefficients.

Let us now discuss the optimality of estimates (2.1). As we shall see in section 5 , the endpoint estimates corresponding to $p=2$ are sharp on spheres. On tori, Bourgain's estimates (1.8) show that intermediate Strichartz estimates are not optimal. It is therefore natural to try to improve these intermediate estimates in some other specific manifolds. This is the aim of our fourth result.

Theorem 4. Assume $(M, g)$ is a riemannian compact manifold of dimension $d \geq 2$, all of whose geodesics are closed with a common period. Then estimate (2.1) can be improved as

$$
\|v\|_{L^{4}(I \times M)} \leq C(I)\left\|v_{0}\right\|_{H^{s}(M)}, \quad s>s_{0}(d),
$$

where $s_{0}(2)=\frac{1}{8}, s_{0}(d)=\frac{d}{4}-\frac{1}{2}$ for $d \geq 3$. Moreover, if $M=S^{d}$ is endowed with canonical metric, then (2.4) is sharp in the sense that similar estimates with $s \leq s_{0}(d)$ (resp. $\left.s<s_{0}(2)\right)$ fail if $d \geq 3$ (resp. $d=2$ ).

The above geometric hypothesis has been studied extensively (see e.g. Besse [1]). Here we use it through a theorem by Guillemin [15] and Colin de Verdière [10] which states that the spectrum of $-\Delta$ is clustered around the sequence $\left((k+\alpha / 4)^{2}\right)_{k \in \mathbb{N}}$ where $\alpha$ is a fixed integer. 
A consequence of estimate (2.4) in three space dimensions is the local wellposedness of the quintic nonlinear Schrödinger equation in $H^{1+\varepsilon}(M)$. A natural open question is of course whether this solution is global in the defocusing case.

In the rest of this note, we discuss briefly the main ingredients in the proof of the above theorems, refering to [7] for more details.

\section{Sketch of the proof of Theorem 1}

As we already observed, the analogue of dispersion estimate (1.6) cannot hold globally on a compact manifold $M$. Moreover, if it would hold for some time $t$, it would imply that $L^{\infty}$ norms of eigenfunctions of the Laplace operator would be estimated by their $L^{1}$ norms, which is known to be false in many cases. This remark suggests that such inequalities have more chance to hold on time intervals which shrink as the frequency of the data is growing. Therefore it is natural to introduce spectral cutoff as follows.

Given $\varphi \in C_{0}^{\infty}(\mathbb{R})$ and $\left.\left.h \in\right] 0,1\right]$, consider the operator $\varphi\left(h^{2} \Delta\right): L^{2}(M) \rightarrow L^{2}(M)$ given by the functional calculus of the Laplace operator. For a given $h>0$, this operator is of course given by a smooth kernel. By standard pseudodifferential methods, one can prove that, as $h$ goes to 0 , this family of operators behaves in every coordinate patch of $M$, as a semiclassical pseudodifferential operator with $C_{0}^{\infty}$ symbol (see e.g. Robert [22] and Helffer-Sjöstrand [16] for related results). As a consequence, we have the following basic estimates.

(i) Spectral Sobolev estimates : if $1 \leq q \leq r \leq \infty$,

$$
\left\|\varphi\left(h^{2} \Delta\right)\right\|_{L^{r}(M)} \leq C h^{\frac{d}{r}-\frac{d}{q}}\left\|\varphi\left(h^{2} \Delta\right)\right\|_{L^{q}(M)} .
$$

(ii) Spectral dyadic inequality. Let $\tilde{\varphi} \in C_{0}^{\infty}(\mathbb{R}), \varphi \in C_{0}^{\infty}(\mathbb{R} \backslash\{0\})$, such that

$$
\tilde{\varphi}(\lambda)+\sum_{k=0}^{\infty} \varphi\left(2^{-2 k} \lambda\right)=1, \quad \lambda \in \mathbb{R}
$$

Then, for $q \in[2, \infty[$,

$$
\|f\|_{L^{q}(M)} \leq C_{q}\|\tilde{\varphi}(\Delta) f\|_{L^{q}(M)}+C_{q}\left(\sum_{k=0}^{\infty}\left\|\varphi\left(2^{-2 k} \Delta\right) f\right\|_{L^{q}(M)}^{2}\right)^{\frac{1}{2}} .
$$

At this stage we can make more precise the remark about dispersion in the beginning of this section. Let $\varphi \in C_{0}^{\infty}(\mathbb{R} \backslash\{0\})$. The operator $\mathrm{e}^{i t \Delta} \varphi\left(h^{2} \Delta\right)$ is represented by the following $C^{\infty}$ kernel

$$
K_{h}(t, x, y)=\sum_{\lambda \in \sigma(\Delta)} \mathrm{e}^{i t \lambda} \varphi\left(h^{2} \lambda\right) e_{\lambda}(x) \overline{e_{\lambda}(y)}
$$


where $\sigma(\Delta)$ denotes the spectrum of $\Delta$ and $\left(e_{\lambda}\right)$ is an orthonormal basis of $L^{2}(M)$ with $\Delta e_{\lambda}=\lambda e_{\lambda}$. Therefore,

$$
\begin{aligned}
\left\|e^{i t \Delta} \varphi\left(h^{2} \Delta\right)\right\|_{L^{1}(M) \rightarrow L^{\infty}(M)} & =\left\|K_{h}(t, \cdot, \cdot)\right\|_{L^{\infty}(M \times M)} \\
& \geq C\left\|K_{h}(t, \cdot, \cdot)\right\|_{L^{2}(M \times M)} \\
& \geq C\left(\sum_{\lambda \in \sigma(\Delta)}\left|\varphi\left(h^{2} \lambda\right)\right|^{2}\right)^{\frac{1}{2}} \geq \frac{C}{h^{\frac{d}{2}}}
\end{aligned}
$$

by the Weyl formula, if $h$ is small enough and $\varphi$ is, say, nonnegative and non identically zero on $]-\infty, 0[$.

The above inequality has two consequences. First, since $\varphi\left(h^{2} \Delta\right)$ is uniformly bounded on $L^{\infty}(M)$, it implies that $\mathrm{e}^{i t \Delta}$ is never bounded from $L^{1}(M)$ to $L^{\infty}(M)$. Hence it is natural to look for modified dispersion estimates of the form

$$
\left\|\mathrm{e}^{i t \Delta} \varphi\left(h^{2} \Delta\right)\right\|_{L^{1}(M) \rightarrow L^{\infty}(M)} \leq \frac{C}{|t|^{\frac{d}{2}}}
$$

as $t$ varies in an interval depending on $h$. Then inequality (3.2) implies that such an interval is necessarily of the form $[-\alpha h, \alpha h]$ for $\alpha$ sufficiently small. The main step in the proof of Theorem 1 is that this information is sharp.

Lemma 3.1. Let $M$ be a compact riemannian manifold of dimension d. Let $\varphi \in$ $C_{0}^{\infty}(\mathbb{R})$. There exists $\alpha>0$ and $C>0$ such that, for every $\left.\left.h \in\right] 0,1\right]$,

$$
\left\|e^{i t \Delta} \varphi\left(h^{2} \Delta\right)\right\|_{L^{1}(M) \rightarrow L^{\infty}(M)} \leq \frac{C}{|t|^{d / 2}}
$$

for every $t \in[-\alpha h, \alpha h]$.

The proof of Lemma 3.1 is based on the following elementary observation : setting $t=h s$ transforms the usual linear Schrödinger equation into the semiclassical one, namely

$$
i h \partial_{s} w+h^{2} \Delta w=0 .
$$

Moreover, the spectral cutoff $\varphi\left(h^{2} \Delta\right)$ allows to assume that the Cauchy data has essentially, in every coordinate patch, a Fourier transform supported in a ball of radius $\mathcal{O}(1 / h)$. As a consequence, one can solve approximately equation (3.4) by the usual WKB ansatz,

$$
\tilde{w}(s, x)=\int_{\mathbb{R}^{d}} e^{\frac{i}{h} \phi(s, x, \xi)} a(s, x, \xi, h) \widehat{w_{0}}\left(\frac{\xi}{h}\right) \frac{d \xi}{(2 \pi h)^{d}}
$$

where

$$
a(s, x, \xi, h)=\sum_{j=0}^{N} h^{j} a_{j}(s, x, \xi) .
$$

and $N$ is to be chosen large enough. The eikonal equation for $\phi$ and the transport equations for the $a_{j}$ 's can be solved locally for any $\xi$ in a compact subset. Moreover, from the construction of $\phi$ one observes that

$$
\phi_{\xi_{i} \xi_{j}}^{\prime \prime}(s, x, \xi)=-2 s g^{i, j}(x)+O\left(s^{2}\right), \quad s \longrightarrow 0,
$$


so that estimate (3.3) follows from the stationary phase formula.

Let us now draw the main steps from Lemma 3.1 to estimates (2.1) of Theorem 1. First we appeal to the following $T T^{*}$ abstract argument.

Proposition 3.2 (Keel-Tao [19]). Let $(X, \mathcal{S}, \mu)$ be a $\sigma$-finite measured space, and $U: \mathbb{R} \mapsto B\left(L^{2}(X, \mathcal{S}, \mu)\right)$ be a weakly measurable map satisfying, for some $A, \sigma>0$,

$$
\|U(t)\|_{L^{2} \rightarrow L^{2}} \leq A, \quad t \in \mathbb{R}
$$

(ii)

$$
\left\|U\left(t_{1}\right) U\left(t_{2}\right)^{\star} f\right\|_{L^{\infty}} \leq \frac{A}{\left|t_{1}-t_{2}\right|^{\sigma}}\|f\|_{L^{1}}, \quad t_{1}, t_{2} \in \mathbb{R} .
$$

Then for every pair $(p, q)$ satisfying

$$
\frac{2}{p}+\frac{2 \sigma}{q}=\sigma, \quad p \geq 2, \quad(p, q) \neq(2, \infty)
$$

one has

$$
\left(\int_{\mathbb{R}}\|U(t) f\|_{L^{q}}^{p} d t\right)^{\frac{1}{p}} \leq B\|f\|_{L^{2}} .
$$

and

$$
\left(\int_{\mathbb{R}}\left\|\int_{-\infty}^{t} U(t) U(\tau)^{*} f(\tau) d \tau\right\|_{L^{q}}^{p} d t\right)^{\frac{1}{p}} \leq C\|f\|_{L^{\overline{p^{\prime}}}\left(\mathbb{R}, L^{\overline{q^{\prime}}}\right)}
$$

for all pairs $(p, q),\left(p^{\prime}, q^{\prime}\right)$ satisfying condition (3.7).

Apply the above proposition to

$$
U(t)=\mathbb{1}_{J}(t) e^{i t \Delta} \varphi\left(h^{2} \Delta\right)
$$

where $J$ is an interval of length $\leq \alpha h$, and $\alpha$ is given by Lemma 3.1. Let us forget for a while estimate (3.9), and focus on (3.8). We obtain

$$
\left(\int_{J}\left\|e^{i t \Delta} \varphi\left(h^{2} \Delta\right) v_{0}\right\|_{L^{q}}^{p} d t\right)^{\frac{1}{p}} \leq C\left\|v_{0}\right\|_{L^{2}}
$$

for all $(p, q)$ satisfying (1.4). Writing $[-1,1]$ as a union of $N$ intervals $J_{k}$ of length $\leq \alpha h$ with $N \lesssim \frac{1}{h}$, we obtain finally

$$
\left(\int_{-1}^{1}\left\|e^{i t \Delta} \varphi\left(h^{2} \Delta\right) v_{0}\right\|_{L^{q}}^{p} d t\right)^{\frac{1}{p}} \leq \frac{\tilde{C}}{h^{1 / p}}\left\|v_{0}\right\|_{L^{2}} .
$$

The proof is completed by combining the above estimate for $h=2^{-k}$ and the spectral dyadic inequality (3.1). 


\section{An analogue of Theorem 1 on $\mathbb{R}^{d}$.}

In this section, we briefly draw how methods used in the proof Theorem 1 can be generalized to Schrödinger equations on $\mathbb{R}^{d}$. Let $g$ be a riemannian metric on $\mathbb{R}^{d}$ satisfying the following uniform bounds

$$
\begin{gathered}
\exists m>0, M>0 ; \quad \forall x \in \mathbb{R}^{d}, \quad m \text { Id } \leq g(x) \leq M \text { Id } \\
\forall \alpha \in \mathbb{N}^{d}, \quad \exists C_{\alpha} ; \quad \forall x \in \mathbb{R}^{d}, \quad\left|\partial^{\alpha} g(x)\right| \leq C_{\alpha}
\end{gathered}
$$

In the case where $g$ is a compactly supported perturbation of the standard metric and if every geodesic of the metric $g$ goes out of any compact set after some time (i.e. $g$ is non-trapping), then it is known that the corresponding linear Schrödinger equation satisfies the same Strichartz estimates as in $\mathbb{R}^{d}$ with the standard metric (see Staffilani and Tataru [27]). Here we can prove the following (weaker) statement, without any geometric assumption on $g$.

Theorem 5. Assume that (4.1) holds. Let $V$ be an unbounded symetric operator on $L^{2}\left(\mathbb{R}^{d}\right)$ with domain $D(V)=H^{1}\left(\mathbb{R}^{d}\right)$ and satisfying

$$
\|V f\|_{L^{2}} \leq C\|f\|_{H^{1}\left(\mathbb{R}^{d}\right)}
$$

Then the solution to the linear Schrödinger equation

$$
\left(i \partial_{t}+\Delta+V\right) u=0, \quad u(0, x)=u_{0}(x)
$$

satisfies the same estimates as in Theorem 1,

$$
\|v\|_{L^{p}\left(I, L^{q}\left(\mathbb{R}^{d}\right)\right)} \leq C(I)\left\|v_{0}\right\|_{H^{1 / p}\left(\mathbb{R}^{d}\right)}
$$

for all $(p, q)$ such that $\frac{2}{p}+\frac{d}{q}=\frac{d}{2}, p \geq 2, q<\infty$, and all finite intervals $I$.

Observe that the abstract perturbation $V$ typically covers potentials associated to $N$-body problems. The selfadjointness of $A=\Delta+V$ is an easy consequence of the Kato-Rellich theorem (see e.g. Reed-Simon [21]).

Let us indicate the main steps of the proof of Theorem 5. First a perturbation argument allows to compare spectral cutoff for $A$ and $\Delta_{g}$, namely for any $\varphi \in$ $C_{0}^{\infty}(\mathbb{R}), 0 \leq r \leq 1,0 \leq s \leq 1$

$$
\left\|\varphi\left(h^{2} A\right)-\varphi\left(h^{2} \Delta_{g}\right)\right\|_{H^{r} \rightarrow H^{s}} \leq C h^{1-s+r} .
$$

Then we prove the modified dispersion estimate in the case $V=0$,

Lemma 4.1. Given $\Psi \in C_{0}^{\infty}\left(\mathbb{R}^{d}\right)$, there exists $\alpha>0, C>0$ such that, for every $h \in] 0,1], t \in J=[-\alpha h, \alpha h]$,

$$
\left\|e^{i t \Delta_{g}} \Psi(h D)\right\|_{L^{1}\left(\mathbb{R}^{d}\right) \rightarrow L^{\infty}\left(\mathbb{R}^{d}\right)} \leq \frac{C}{|t|^{d / 2}}
$$


Indeed, although the WKB method is used in Lemma 3.1 locally with respect to the $x$ variable, here, according to assumptions (4.1), the solutions to elkonal and transport equations are defined globally on $[-\alpha, \alpha] \times \mathbb{R}^{d}$.

Applying Proposition 3.2 to $U(t)=\Psi(h D) \mathrm{e}^{i t \Delta_{g}}$, we obtain, if $|J| \leq \alpha h$,

$$
\left(\int_{J}\left\|\Psi(h D) \mathrm{e}^{i t \Delta_{g}} v_{0}\right\|_{L^{q}}^{p}\right)^{1 / p} \leq C\left\|v_{0}\right\|_{L^{2}}
$$

Assume $\Psi \in C_{0}^{\infty}\left(\mathbb{R}^{d} \backslash\{0\}\right)$; comparing the action of $\Psi(h D)$ with the action of a suitable spectral cutoff $\varphi\left(h^{2} \Delta_{g}\right)$ for $\varphi \in C_{0}^{\infty}(\mathbb{R} \backslash\{0\})$, we infer

$$
\left(\int_{J}\left\|\Psi(h D) \mathrm{e}^{i t \Delta_{g}} v_{0}\right\|_{L^{q}}^{p} d t\right)^{1 / p} \leq C\left\|\varphi\left(h^{2} \Delta\right) v_{0}\right\|_{L^{2}}+C_{N} h^{N}\left\|v_{0}\right\|_{L^{2}} .
$$

As a third step, we are going to take advantage of the conservation of the $L^{2}$ norm

$$
\left\|e^{i t A} u_{0}\right\|_{L^{2}}=\left\|u_{0}\right\|_{L^{2}}
$$

to treat the perturbation as a source term: write the solution of equation (4.3) as

$$
u(t)=e^{i t \Delta_{g}} u_{0}-\int_{0}^{t} e^{i t(t-\tau) \Delta_{g}}(V u(\tau)) d \tau .
$$

Using estimates (4.8) we obtain, if $J=[0, \alpha h]$,

$$
\|\Psi(h D) u\|_{L^{p}\left(J, L^{q}\right)} \leq C\left\|u_{0}\right\|_{L^{2}}+C\left\|\varphi\left(h^{2} \Delta_{g}\right)(V u)\right\|_{L^{1}\left(J, L^{2}\right)}+C_{N} h^{N}\|V u\|_{L^{1}\left(J, L^{2}\right)}
$$

Since $\varphi$ is compactly supported,

$$
\left\|\varphi\left(h^{2} \Delta_{g}\right)(V f)\right\|_{L^{2}} \leq \frac{1}{h}\left\|\varphi\left(h^{2} \Delta_{g}\right)(V f)\right\|_{H^{-1}} \leq \frac{C}{h}\|f\|_{L^{2}}
$$

since $V$ is bounded from $L^{2}$ to $H^{-1}$ by duality.

Taking advantage of the length of $J$, we conclude

$$
\|\Psi(h D) u\|_{L^{p}\left(J, L^{q}\right)} \leq C\left\|u_{0}\right\|+C_{N} h^{N}\left\|u_{0}\right\|_{H^{1}}
$$

Next we apply the above estimate to $\varphi\left(h^{2} A\right) u_{0}$ in place of $u_{0}$. Then $u$ is replaced by $\varphi\left(h^{2} A\right) u$, and since

$$
\left\|\varphi\left(h^{2} A\right) u_{0}\right\|_{H^{1}} \leq \frac{C}{h}\left\|\varphi\left(h^{2} A\right) u_{0}\right\|_{L^{2}}
$$

we obtain

$$
\left\|\Psi(h D) \varphi\left(h^{2} A\right) u\right\|_{L^{p}\left(J, L^{q}\right)} \leq C\left\|\varphi\left(h^{2} A\right) u_{0}\right\|_{L^{2}} .
$$

Then we sum on intervals $J$ of length $|J| \leq \alpha h$ to get an estimate on intervals of fixed length

$$
\left\|\Psi(h D) \varphi\left(h^{2} A\right) u\right\|_{L^{p}\left(I, L^{q}\right)} \leq C h^{1 / p}\left\|\varphi\left(h^{2} A\right) u_{0}\right\|_{L^{2}} \leq C\left\|\varphi\left(h^{2} A\right) u_{0}\right\|_{H^{1 / p}} .
$$


It remains to sum on frequencies. Observe that

$$
\Psi(h D)=\Psi(h D) \varphi\left(h^{2} A\right)+\Psi(h D)\left(1-\varphi\left(h^{2} \Delta\right)\right)+\Psi(h D)\left(\varphi\left(h^{2} \Delta\right)-\varphi\left(h^{2} A\right)\right)
$$

The second term in the right hand side is small due to the choice of $\varphi$. For the third term we apply estimate (4.5) with $s=\frac{2}{p}=d\left(\frac{1}{2}-\frac{1}{q}\right)$ and $r=\frac{1}{p}$, and we conclude

$$
\|\Psi(h D) u\|_{L^{p}\left(I, L^{q}\right)} \leq C\left\|\varphi\left(h^{2} A\right) u_{0}\right\|_{H^{1 / p}}+C h^{1-\frac{1}{p}}\left\|u_{0}\right\|_{H^{1 / p}} .
$$

Therefore the theorem follows by summing on frequencies according to the usual dyadic $L^{q}$ inequality.

\section{The nonlinear problems.}

First we observe that estimates (2.1) in Theorem 1 yield classically estimates for nonhomogeneous Schrödinger equation.

Corollary 5.1. If $p, q$ satisfy $\frac{2}{p}+\frac{d}{q}=\frac{d}{2}, p \geq 2$ and $q<\infty$,

$$
\left\|\int_{0}^{t} e^{i(t-\tau) \Delta} f(\tau) d \tau\right\|_{L^{p}\left([0, T], L^{q}(M)\right)} \leq C_{T}\|f\|_{L^{1}\left([0, T], H^{\frac{1}{p}}(M)\right)} .
$$

From this we deduce easily the following general local wellposedness result below the energy threshold.

Proposition 5.2. Let $F$ be a polynomial of degree $\beta \geq 2$ with $F(0)=0$, and

$$
s>\frac{d}{2}-\frac{1}{\beta-1}, s>\frac{d-1}{2} .
$$

For every $u_{0} \in H^{s}(M)$, there exists $T>0$ and a unique solution

$$
u \in C\left([-T, T], H^{s}(M)\right) \cap L^{p}\left([-T, T], L^{\infty}(M)\right)
$$

of the equation

$$
i \partial_{t} u+\Delta u=F(u), \quad u(0, x)=u_{0}(x)
$$

with $t \in \mathbb{R}, x \in M$, and where $F$ is a (nonlinear) polynomial, $F(0)=0$ for some $p>\beta-1$. Moreover

(i) If $\left\|u_{0}\right\|_{H^{s}}$ is bounded, then $T$ is bounded from below by a positive constant.

(ii) If $u_{0} \in H^{r}$ for some $r>s, u \in C\left([-T, T], H^{r}(M)\right)$.

The proof is a standard contraction principle in a suitable Banach space, which is selected upon the following constraints. Since $F$ has degree $\beta$,

$$
\|F(u)\|_{H^{s}} \leq C\left(1+\|u\|_{L^{\infty}}\right)^{\beta-1}\|u\|_{H^{s}}
$$

for every $s \geq 0$. As a consequence we need to control $u$ in $L_{t}^{p} L_{x}^{\infty}$ for some $p>\beta-1$, where the strict inequality guarantees contraction. If moreover $p \geq 2$, this will be 
achieved through the $L_{t}^{p} L_{x}^{q}$ estimate (5.1) combined with the Sobolev imbedding $W^{\sigma, q} \subset L^{\infty}$, with $\sigma>d / q=d / 2-2 / p$. Since the $L_{t}^{p} W_{x}^{\sigma, q}$ norm is controlled by the $H^{s}$ norm of the data with $s=\sigma+1 / p>d / 2-1 / p$, the natural Banach space is therefore

$$
Y_{T}=C\left([-T, T], H^{s}(M)\right) \cap L^{p}\left([-T, T], W^{\sigma, q}(M)\right) .
$$

Theorem 2 in two space dimensions can be easily deduced from Proposition 5.2, since the regularity $s$ can be chosen $<1$ for all degree $\beta$. One can therefore combine local wellposedness with conservation laws (1.2) and (1.3).

In three space dimensions, the situation is more intricate. Indeed, the gain of derivative provided by Theorem 1 is $1 / p \leq 1 / 2$ derivative. On the other hand, assuming a control of the $H^{1}$ norm, Sobolev inequality imposes us to gain at least $1 / 2$ derivative in order to handle nonlinear terms. As a consequence, we are forced to use endpoint Strichartz estimates, which correspond to $p=2$. Moreover, in this situation, Proposition 5.2 above only yields local wellposedness in $H^{1+\varepsilon}$ for cubic nonlinearities. In order to get global existence in Theorem 3, we shall rather appeal to the nonhomogeneous estimate on a small interval which is directly issued from Proposition 3.2, namely, for any $\varphi \in C_{0}^{\infty}(\mathbb{R})$, there exists $C>0$ such that for any $h \in] 0,1], f$ supported in $J$,

$$
\left(\int_{J}\left\|\int_{-\infty}^{t} e^{i(t-\tau) \Delta} \varphi\left(h^{2} \Delta\right) f(\tau) d \tau\right\|_{L^{6}}^{2} d t\right)^{\frac{1}{2}} \leq C\left\|\varphi\left(h^{2} \Delta\right) f\right\|_{L^{2}\left(J, L^{\frac{6}{5}}\right)}
$$

if $|J| \leq \frac{\alpha}{2} h$. Applying this inequality to the quadratic equation

$$
i \partial_{t} u+\Delta u=P^{\prime}\left(|u|^{2}\right) u
$$

where $P \in S^{3 / 2}$, and summing on $N \leq C / h$ intervals, we obtain, after some work, finally

$$
\sum_{k=0}^{\infty}\left\|\varphi\left(2^{-2 k} \Delta\right) u\right\|_{L^{2}\left(I, L^{\infty}\right)}^{2} \leq C\left(1+\|u\|_{L^{\infty}\left(I, H^{1}\right)}^{2}\right)^{2}
$$

for any finite interval $I$, where $C$ is bounded if the length of $I$ is bounded. Observe that the right hand side of (5.5) is controlled by conservation laws. As a consequence, we derive the following logarithmic inequality,

$$
\|u\|_{L^{2}\left([0, T], L^{\infty}\right)} \leq C\left(\log \left(2+\|u\|_{L^{2}\left([0, T], H^{2}\right)}\right)\right)^{\frac{1}{2}}
$$

Finally we come back to equation (5.4) and we apply the standard propagation estimates in $L^{2}$,

$$
\|u(T)\|_{H^{2}} \leq\left\|u_{0}\right\|_{H^{2}}+C\left(\log \left(2+\|u\|_{L^{2}\left([0, T], H^{2}\right)}\right)\right)^{\frac{1}{2}}\|u\|_{L^{2}\left([0, T], H^{2}\right)}
$$

Taking the square of the later inequality and integrating on $[0, s]$ we observe that $F(s)=\|u\|_{L^{2}\left([0, s], H^{2}\right)}^{2}$ satisfies

$$
F(s) \leq C\left(1+\int_{0}^{s} F(t) \log (2+F(t)) d t\right)
$$

hence is bounded if $s$ is bounded. Coming back to (5.7), this completes the proof. Remark 5.3. Of course the above arguments apply as well to nonlinear perturbations of problems on $\mathbb{R}^{3}$ studied in section 4 . 


\section{On the optimality of Strichartz estimates}

On compact manifolds it is natural to test the optimality of Strichartz estimates on eigenfunctions of the Laplace operator. Indeed, if $\Delta v_{0}=\lambda v_{0}$, then

$$
v(t)=\mathrm{e}^{i t \Delta} v_{0}=\mathrm{e}^{i t \lambda} v_{0}
$$

thus $\|v\|_{L^{p}\left(I, L^{q}(M)\right.}=|I|^{1 / p}\left\|v_{0}\right\|_{L^{q}(M)}$ while $\left\|v_{0}\right\|_{H^{s}(M)} \sim \lambda^{s / 2}\left\|v_{0}\right\|_{L^{2}(M)}$ if $s \geq 0$ and $\lambda$ is large. Thus we are led to discuss the growth of $L^{q}$ norms $(q>2)$ of eigenfunctions with respect to their $L^{2}$ norms. In this context one disposes of the following general result.

Proposition 6.1 (Sogge [24], [25]). For all $k \geq 1$, denote by $\Pi_{k}$ the spectral projector

$$
\Pi_{k}=\mathbb{1}_{-\Delta \in\left[k^{2},(k+1)^{2}\right]},
$$

where $\Delta$ is the Laplace-Beltrami operator on a compact riemannian d-manifold $M$, $d \geq 2$. We have

$$
\left\|\Pi_{k}\right\|_{L^{2}(M) \rightarrow L^{q}(M)} \leq C k^{s(q)}
$$

where

$$
s(q)= \begin{cases}\frac{d-1}{2}\left(\frac{1}{2}-\frac{1}{q}\right), & \text { if } \quad 2 \leq q \leq \frac{2(d+1)}{d-1}, \\ \frac{d-1}{2}-\frac{d}{q}, & \text { if } \quad \frac{2(d+1)}{d-1} \leq q \leq \infty .\end{cases}
$$

Moreover, these estimates are sharp.

In particular, the above estimates give bounds on the growth of $L^{q}$ norms of eigenfunctions normalized in $L^{2}$. However the sharpness of these bounds is a much more difficult problem (see Sogge-Zelditch [26] for recent results in this direction), except in specific geometries. For instance, if $M=S^{d}$, for all $q \geq 2$ one can find a sequence $\left(h_{k}\right)$ of spherical harmonics, such that

$$
\left\|h_{k}\right\|_{L^{q}} \approx c(q) k^{s(q)}\left\|h_{k}\right\|_{L^{2}}, \quad k \longrightarrow \infty
$$

where $s(q)$ is the exponent in estimate (6.1) (see [23]). If $d \geq 3$, observe that $s\left(2^{*}\right)=1 / 2$. Therefore estimate (6.2) implies that the endpoint Strichartz estimates of Theorem $1\left(p=2, q=2^{*}, s=1 / 2\right)$ are optimal on $S^{d}$ for $d \geq 3$. Similarly, in the case $d=2$, letting $p$ tend to 2 , the argument gives the optimality of the $L^{p} L^{q}$ estimate on $S^{2}$ up to the loss of $\varepsilon$ derivatives.

Finally we turn to intermediate Strichartz estimates corresponding to $q=4$ as in Theorem 4. Rather than giving a complete proof of Theorem 4, for which we refer to [7], we shall discuss in detail the case of $M=S^{3}$. In this case, Sogge's estimate for $q=4$ reads

$$
\left\|f_{k}\right\|_{L^{4}} \leq C k^{\frac{1}{4}}\left\|f_{k}\right\|_{L^{2}}, \quad k \rightarrow \infty
$$

for spherical harmonics of degree $k$, and optimality of the above estimates is attained for instance by zonal spherical harmonics, which in this particular dimension have the following simple expression,

$$
h_{k}(x)=\frac{\sin ((k+1) \theta)}{\sin \theta}
$$


where $\theta \in[0, \pi], \cos \theta=\left\langle x, x_{0}\right\rangle$, for a chosen point $x_{0} \in S^{d}$. Notice that the volume element in variable $\theta$ becomes $\sin ^{2} \theta d \theta$ (up to a multiplicative constant), so that $\left\|h_{k}\right\|_{L^{2}} \approx 1$ and (6.2) can be easily checked for $q \geq 4$.

First let us prove the estimate of Theorem 4 in this case. A solution of the linear Schrödinger equation reads

$$
v(t, x)=\sum_{k=0}^{\infty} \mathrm{e}^{i t\left(1-(k+1)^{2}\right)} f_{k}(x)
$$

where $f_{k}$ are spherical harmonics of degree $k$. Observe that $v$ is $2 \pi$-periodic in time. Therefore the inequality we have to prove is

$$
\|v\|_{L^{4}\left(\mathbb{T} \times S^{3}\right)} \leq C_{s}\|v(0)\|_{H^{s}\left(S^{3}\right)}
$$

for all $s>1 / 4$. By dyadic decomposition, we may assume that $N \leq k \leq 2 N$, so that (6.3) is equivalent to

$$
\|v\|_{L^{4}\left(\mathbb{T} \times S^{3}\right)} \leq C N^{s}\left(\sum_{k}\left\|f_{k}\right\|_{L^{2}\left(S^{3}\right)}^{2}\right)^{\frac{1}{2}} .
$$

But the above inequality is an easy consequence of the following computation,

$$
\begin{aligned}
\|v\|_{L^{4}\left(\mathbb{T} \times S^{3}\right)}^{4}=\left\|v^{2}\right\|_{L^{2}\left(\mathbb{T} \times S^{3}\right)}^{2} & =\sum_{2 N^{2} \leq \tau \leq 9 N^{2}}\left\|\sum_{(k, l):(k+1)^{2}+(l+1)^{2}=\tau} f_{k} f_{l}\right\|^{2} \\
& \leq \sup _{2 N^{2} \leq \tau \leq 9 N^{2}} r_{2}(\tau)\left(\sum_{k}\left\|f_{k}\right\|_{L^{4}}^{2}\right)^{2}
\end{aligned}
$$

where $r_{2}(\tau)=\#\left\{(k, l):(k+1)^{2}+(l+1)^{2}=\tau\right\}=\mathcal{O}\left(N^{\varepsilon}\right)$ (see e.g. Grosswald[14]). Using Sogge's estimate, we infer (6.3) for $s>1 / 4$.

Finally we prove that estimate (6.3) fails for $s=1 / 4$. As in Bourgain [2] the argument is based on Gauss sums. We start from the following estimate,

$$
\left\|\sum_{n \in \mathbb{Z}} \mathrm{e}^{-\frac{2 i \pi}{p} a n^{2}} \psi\left(\frac{n}{N}\right)\left|-\frac{N}{\sqrt{p}}\right| \int_{\mathbb{R}} \psi(y) d y\right\| \leq p \int_{\mathbb{R}}\left|\psi^{\prime}(y)\right| d y,
$$

where $\psi$ is $C^{1}$ and compactly supported on $\mathbb{R}, N \in \mathbb{N}, p$ is a prime odd number, $a \in\{1, \ldots, p-1\}$. This estimate is an easy combination of the celebrated Gauss identity

$$
\left|\sum_{r=0}^{p-1} \mathrm{e}^{-\frac{2 i \pi}{p} a n^{2}}\right|=\sqrt{p}
$$

and of the elementary inequality

$$
\left|\sum_{q \in \mathbb{Z}} \psi\left(\frac{p q+r}{N}\right)-\frac{N}{p} \int_{\mathbb{R}} \psi(y) d y\right| \leq p \int_{\mathbb{R}}\left|\psi^{\prime}(y)\right| d y .
$$


Then we define the following solution of the Schrödinger equation on $S^{3}$,

$$
v_{N}(t, \theta)=\sum_{n=1}^{\infty} \mathrm{e}^{i t\left(1-n^{2}\right)} \chi\left(\frac{n}{N}\right) \frac{\sin (n \theta)}{\sin \theta},
$$

where $\chi$ is $C^{1}$, nonnegative, not identically zero, and compactly supported in $] 0,+\infty[$. We claim that there exits some $\delta>0$ such that, for all $N$ large enough, for all odd prime number $p$ such that $p<<N^{2 / 3}$, for all $a \in\{1, \ldots, p-1\}$,

$$
\left|v_{N}(t, \theta)\right| \geq \frac{N^{2}}{2 \sqrt{p}}\left|\int_{0}^{\infty} y \chi(y) d y\right|
$$

on the set

$$
\Omega_{a, p}=\left\{(t, x):\left|t-\frac{2 \pi a}{p}\right| \leq \frac{\delta}{N^{2}},|\theta| \leq \frac{\delta}{N}\right\} .
$$

Indeed, write $t=\frac{2 \pi a}{p}+\frac{s}{N^{2}}, \theta=\frac{\gamma}{N}$ with $|s| \leq \delta$ and $|\gamma| \leq \delta$. Then

$$
v_{N}(t, \theta)=\frac{\mathrm{e}^{i t}}{\sin (\gamma / N)} \sum_{n} \mathrm{e}^{-\frac{2 i \pi}{p}\left(a n^{2}\right)} \psi\left(\frac{n}{N}, s, \gamma\right)
$$

with

$$
\psi(y, s, \gamma)=\chi(y) \mathrm{e}^{-i s y^{2}} \sin (\gamma y)
$$

Observe that

$$
\frac{1}{\sin (\gamma / N)} \int_{0}^{\infty} \psi(y, s, \gamma) d y \sim N \int_{0}^{\infty} y \chi(y) d y
$$

as $N$ tend to $\infty$ and $s, \gamma$ tend to 0 , with similar estimates on the integral of $\left|\psi^{\prime}\right|$. Thus estimate (6.5) is a consequence of inequality (6.4). It remains to notice that sets $\Omega_{a, p}$ are all disjoint as $p$ varies among odd prime numbers much smaller than $N$, so that

$$
\left\|v_{N}\right\|_{L^{4}\left(\mathbb{T} \times S^{3}\right)}^{4} \geq C \sum_{p<<N^{2 / 3}} \frac{N^{8}}{p^{2}} p \frac{1}{N^{2}} \frac{1}{N^{3}}=C N^{3} \sum_{p<<N^{2 / 3}} \frac{1}{p}
$$

while $\left\|v_{N}\right\|_{H^{1 / 4}\left(S^{3}\right)}^{2} \approx N^{3 / 2}$. Thus the divergence of $\sum_{p} \frac{1}{p}$ implies that estimate (6.3) fails for $s=1 / 4$.

Remark 6.2. 1. The proof of estimates (2.4) in Theorem 4 is an adaptation of the above argument, using the clustering property of the spectrum of the Laplacian and the full strength of Sogge's estimates (6.1), see [7]. The proof of optimality for $d \geq 3$ also proceeds the same way as above, using Gauss sums, but is more technical.

2. In three space dimensions, $2(d+1) /(d-1)=4$, thus estimates (2.4) and (2.1) for $p=2$ imply by interpolation

$$
\|v\|_{L_{t}^{p} L_{x}^{q}} \leq C\left\|v_{0}\right\|_{H^{s}}, \quad s>s(q)
$$

for every $(p, q)$ satisfying (1.4). Observe that, by the information (6.2) on eigenfunctions, we know that these estimates are optimal on $S^{3}$ (apart from $\varepsilon$ derivative) with respect to $(q, s)$. It would be interesting to know whether such estimates can be generalized to other manifolds, or if a bad distribution of eigenvalues can be combined with concentration of eigenfunctions to yield a higher loss of derivative. 


\section{References}

[1] A. Besse, Manifolds all of whose geodesics are closed, Springer-Verlag, Berlin-New York, 1978.

[2] J. Bourgain, Fourier transform restriction phenomena for certain lattice subsets and application to nonlinear evolution equations I. Schrödinger equations, Geom. and Funct. Anal. 3 (1993), 107-156.

[3] J. Bourgain, Exponential sums and nonlinear Schrödinger equations, Geom. and Funct. Anal. 3 (1993), 157-178.

[4] J. Bourgain, Global solutions of nonlinear Schrödinger equations, Colloq. Publications, American Math. Soc., 1999.

[5] J. Bourgain, Global wellposedness of defocusing critical nonlinear Schrödinger equations in the radial case, J. Amer. Math. Soc. 12 (1999), $145-171$.

[6] H. Brézis, T. Gallouët, Nonlinear Schrödinger evolution equations, Nonlinear Analysis, Theory, Methods and Applications, 4 (1980), 677681.

[7] N. Burq, P. Gérard, N. Tzvetkov, Strichartz inequalities and the nonlinear Schrödinger equation on compact manifolds, preprint 2001.

[8] T. Cazenave, An introduction to nonlinear Schrödinger equations, Text. Met. Mat. 22, Inst. Mat., Rio de Janeiro, 1989.

[9] T. Cazenave, F. Weissler, The Cauchy problem for the critical nonlinear Schrödinger equation in $H^{s}$, Nonlinear Analysis, Theory, Methods and Applications, (1990), 807-836

[10] Y. Colin de Verdière, Le spectre des opérateurs elliptiques à bicaractéristiques toutes periodiques, Comment. Math. Helvetici 54 (1979), 508-522.

[11] E. B. Davies, Spectral theory and differential operators, Cambridge University Press (1995).

[12] J. Ginibre, G. Velo, The global Cauchy problem for the nonlinear Schrödinger equation, Ann. I.H.P. (Anal. non lin.) 2 (1985), 309-327.

[13] J. Ginibre and G. Velo, Smoothing properties and retarded estimates for some dispersive evolution equations, Commun. Math. Phys. 144 (1992), $163-188$.

[14] E. Grosswald, Representations of Integers as Sums of Squares, Springer-Verlag, 1985. 
[15] V. Guillemin, Lectures on spectral theory of elliptic operators, Duke Math. J. 44 (1977), 129-137.

[16] B. Helffer, J. Sjöstrand, Equation de Schrödinger avec champ magnetique et équation de Harper, Lecture notes in Physics, 345 (1989), 118-197.

[17] L. Kapitanskii, Some generalizations of the Strichartz-B-Brenner inequality, Leningrad Math. J. 1 (1990), 693-726.

[18] T. Kato, On nonlinear Schrödinger equations, Ann. I.H.P. (Phys. Théor.) 46 (1987), 113-129.

[19] M. Keel, T. Tao, Endpoint Strichartz estimates, Amer. J. Math. 120 (1998), 955-980.

[20] G. Lebeau, Contrôle de l'équation de Schrödinger, J. Math. Pures Appl. 71 (1992), 267-291.

[21] M. Reed, B. Simon, Methods of Modern Mathematical Physics, vol.2, Academic Press, 1975.

[22] D. Robert Autour de l'approximation semi-classique, Progress in Mathematics, vol. 68, Birkhäuser, 1987.

[23] C. D. Sogge, Oscillatory integrals and spherical harmonics, Duke Math. J. 53 (1986), 43-65.

[24] C. D. Sogge, Concerning the $L^{p}$ norm of spectral clusters for second order elliptic operators on compact manifolds, J. Funct. Anal. 77 (1988), 123-138.

[25] C. D. Sogge, Fourier integrals in classical analysis, Cambridge tracts in Mathematics, 1993.

[26] C. D. Sogge, S. Zelditch Riemannian manifolds with maximal eigenfunction growth, Preprint 2001.

[27] G. Staffilani, D. Tataru, Strichartz estimates for a Schrödinger operator with nonsmooth coefficients, Preprint 2000.

[28] R. Strichartz, Restriction of Fourier transforms to quadratic surfaces and decay of solutions of wave equations, Duke Math. J. 44 (1977), 705-714.

[29] P. A. Tomas, A restriction theorem for the Fourier transform, Bull. Amer. Math. Soc. 81 (1975), 477-478.

[30] K. Yajima, Existence of solutions for Schrödinger evolution equations, Commun. Math. Phys. 110 (1987), 415-426. 
Université de Paris-Sud, Mathématiques, BÂtiment 425,

91405 ORSAY. FRANCE

Nicolas.Burq@math.u-psud.fr

Patrick.Gerard@math.u-psud.fr

Nikolay.Tzvetkov@math.u-psud.fr 